\title{
NOVITATES BURSERACEARUM III
}

\section{J. J. SWART}

\section{(Botanical Museum and Herbarium, Utrecht)}

(received September 22nd, 1965)

Protium aracouchini (Aubl.) March. var. angustifolium Swart n. var. Folia 1- usque ad 5-juga; interjugum basale petiolo plerumque longius sed interjugis aliis brevius; petioluli breves; foliola oblongolanceolata $7.5-10 \mathrm{~cm}$ longa, 2-2.75 cm lata, apicem versus distincte angustata, apice gradatim in acumen lineare sexies usque ad nonies longius quam latius acuminato; nervi sec. utrinque usque ad 20.

Holotypus: Lindeman 4504 (U, 078268B).

SuRINAM, south of Paramaribo, near Lelydorp, Lindeman 4504 (Aug. 1953) (U); between Suriname-river and Mapanecreek, Lindeman 4808 (Oct. 1953) (U); idem, Lindeman 4925 (Oct. 1953) (U).

British Gulana, Bartica-Potaro road, For. Dept. Br. Guiana 5608 (Mar. 1948) $(\mathrm{K}, \mathrm{U})$.

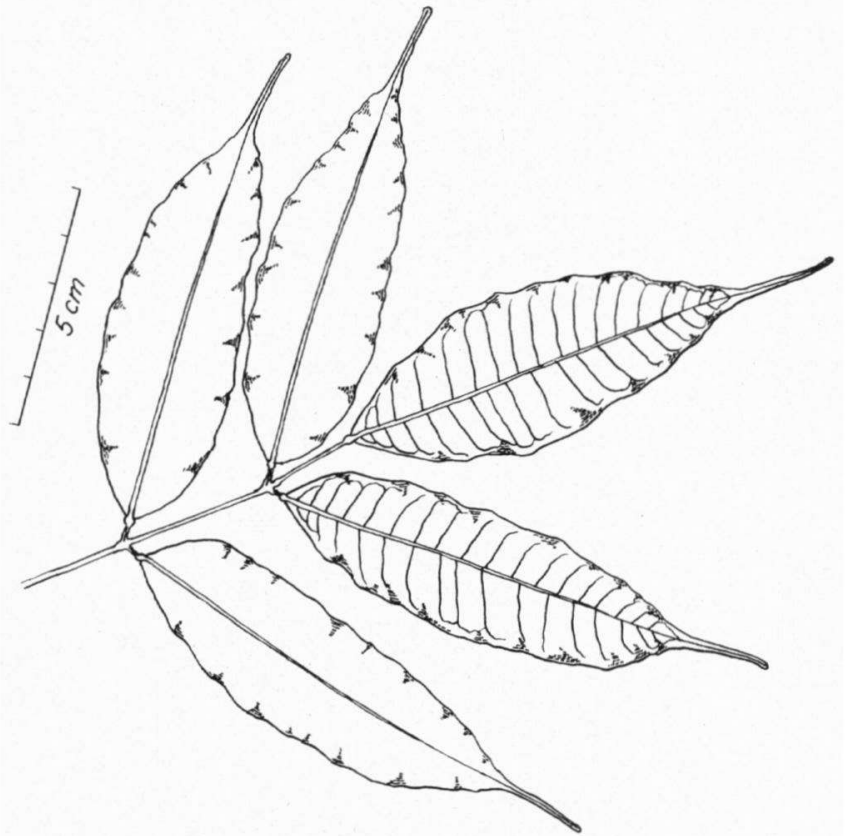

Fig. 1.

$$
\text { Swart-Lindemán } 4504 \text { (U). }
$$

Protium copal (Schlecht. et Cham.) Engl. var. matudea Swart n. var. Folia magna usque ad $50 \mathrm{~cm}$ longa, 4-juga; foliolis terminalibus 
oblongis, non obovatis. Rhachis ramulisque inflorescentiae cum calyce et fructibus glabrescentes:

Holotypus: Matuda 3414 (K).

Mexico, Retiro, Tenosique, Matuda 3414 (Jun. 1939) (K); Rivina, Palenque, Matuda 3698 (Jul. 1939) (K).

Protium demerarense Swart n. sp. Arbor magna, usque as $24 \mathrm{~m}$ alta, $30-40 \mathrm{~cm}$ diam. Ramuli gracilioris angulosi glabri cinerei vel fusci lenticellis parvis brunneis obtectis. Folia 3-juga raro 1- aut 2-juga 20-30 (15-33) cm longa, petiolis semiteretibus basi subincrassatis $3.5-4.5 \mathrm{~cm}$ longis cum interjugis petiolulisque brevissime densiuscule pilosis, interjugis teretibus carinatis utrinque subincrassatis $3.5-4.5 \mathrm{~cm}$ longis, petiolulis semiteretibus vadoso-sulcatis utrinque incrassatis $\mathbf{0 . 7 5}$ $(0.5-1.5) \mathrm{cm}$ longis sed foliolorum terminalium teretibus carinatis $2.5-3(2-4) \mathrm{cm}$ longis, foliolis oblongis $9-14 \mathrm{~cm}$ longis $3-4.5 \mathrm{~cm}$ latis terminalibus prope basim angustatis lateralibus interdum apicem versus paulo angustatis, apice abruptius acuminatis acumine sublineari obtuso quinquies longiore quam latiore $10-15 \mathrm{~mm}$ longo $2-3 \mathrm{~mm}$ lato, basi cuneato, margine integro sed in sementibus prope apicem serratis, subcoriaceis glabris supra nitidis infra opacis, nervis lateralibus utrinque circ. 12 nervis, prim. facie superiore brevissime densiuscule pilosis, nervis ceteris totis glabris. Inflorescentiae axillares thyrsoideae e basi multiramosae fere glomeruliformes, usque ad 2.75 longae. Rhaches teretes striatae robustae cum bracteis bractolisque $0.5 \mathrm{~mm}$ longis pedicelisque sparse puberulae. Pedicelli teretes striati flori subaequilongi. Flores 5-meri $4 \mathrm{~mm}$ longi. Galyx cupuliformis circ. $1 \mathrm{~mm}$ altus glaber aut pilis nonnullis sparsis vestitus lobis semiorbicularibus subacuminatis tubo aequilongis. Petala subinduplicativo - valvata oblongo-lanceolata triangularia acuta apiculo incrassato inflexo. Stamina 10 in $\mathrm{fl}$. masc. $2 \mathrm{~mm}$ longa filamenta subulata antheris oblongis triplo longiora. Discus annularis 10-lobus glaber circ. $0.25 \mathrm{~mm}$ altus. Pistillum in $\mathrm{fl}$. masc. basi disco cinctum $0.75 \mathrm{~mm}$ altum ex ovario applanato-globoso 5-lobo 5-loculari pilis nonnullis adpressis sparso et stigmate sessili vel subsessili 5-lobo compositum. Drupa obliquooblonga utrinque subacuta $3.5 \mathrm{~cm}$ longa $1.5 \mathrm{~cm}$ diam. semper monoloculari monopyrena epicarpio lenticellis ellipticis numerosis obtecto mesocarpio tenui carnoso endocarpio tenui lignoso fragili.

Holotypus: For. Dept. Br. Guinea 3479 (U, 33160B).

British Guiana, Mahdia Creek, Potaro-river, at $\pm 100 \mathrm{~m}$, Bartica-Potaro road, For. Dep. Br. Guiana 3479 (Jun. 1942, fl. masc.) (K, U); idem, For. Dep. Br. Guiana 3767 (Jan. 1943, fr.) (K, U); idem, For. Dep. Br. Guiana 3796 (Jan. 1943, fr.) (K); idem, For. Dep. Br. Guiana 4217 (1943, seedlings) (K, U).

Nom. vern.: "purokai".

This species resembles Protium altsonii Sandw. and $P$. hostmannii (Miq.) Engl., but the former species differs by its 4- to 7-jugate leaves bearing lanceolate to lanceolate-oblong leaflets with 14 to 17 pairs of sec. nerves, its smaller flowers with densely pilose ovary and its much smaller drupes. The latter differs by its usually 2-jugate 
much shorter leaves, composed of a petiole shorter than the interjuga and of shorter, more elliptic leaflets with 10 pairs of sec. nerves and an acumen only 2- to 3-times as long as wide, by its 4-merous, much smaller flowers with a rather densely pilose ovary and by half-sized fruits.

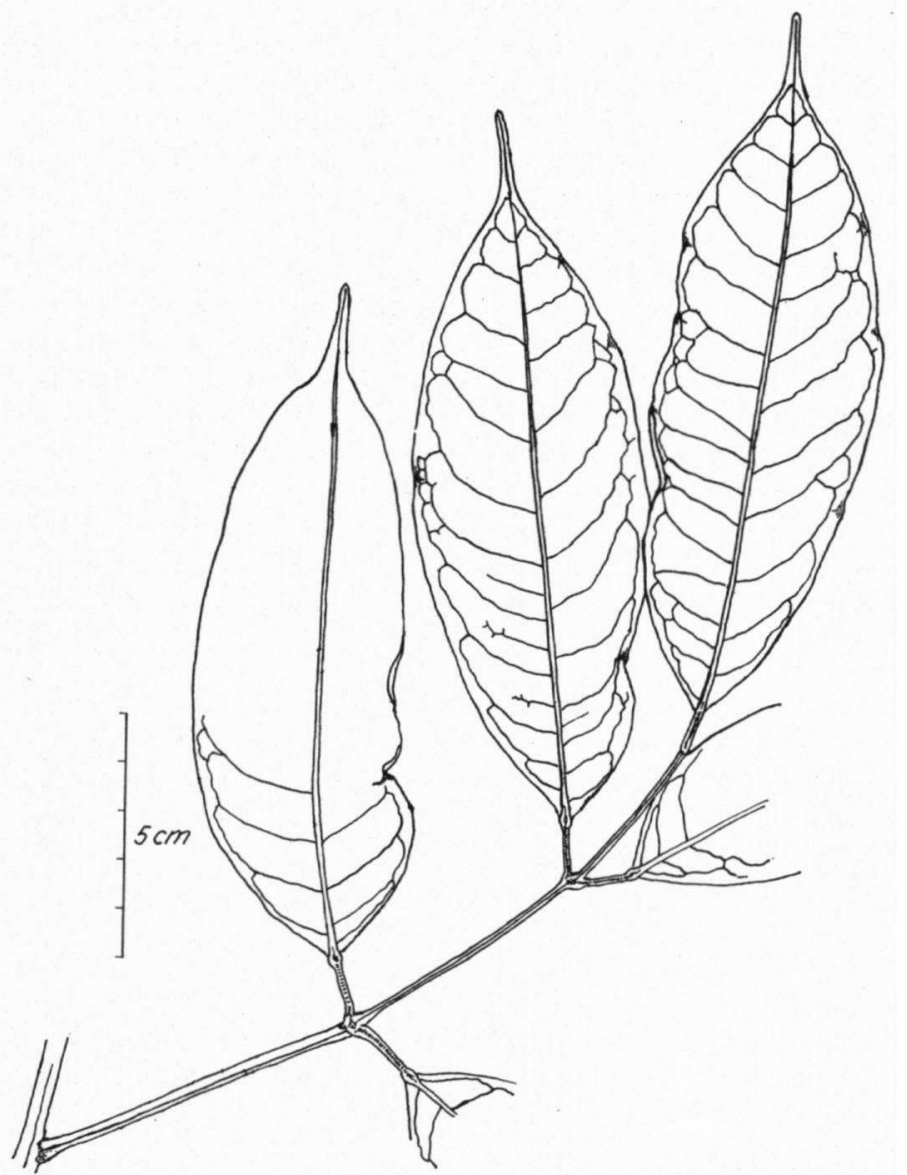

Fig. 2. Protium demerarense Swart - For. Dept. Br. Guiana 3479 (U).

Protium divaricatum Eng. var. trijugum Swart n. var. Folia 3-juga $25-30 \mathrm{~cm}$ longa, petiolis $4-7 \mathrm{~cm}$ longis, interjugis terete-carinatis $2-5 \mathrm{~cm}$ longis, nervis sec. foliolorum utrinque $10-11$. Inflorescentiae interdum folio suffulcienti fere aequilongae minime $2 / 3$ partes eorum aequantes. Rhachis ramuli et pedicellorum partes inferiores sparse puberuli.

Holotypus: Black 47-1507 (IAN).

BraziL, Amazonia, near Tefé, Black 47-1507 (Sep. 1947. fl. masc.) (IAN); id., id., Black 47-1529 (Sep. 1947, fl. masc.) (IAN).

Protium dusenii Swart n. sp. Arbor. Ramuli graciles teretes 
striati glabri fusci. Folia 2- vel 3-juga $17(14-23) \mathrm{cm}$ longa ubique glabra, petiolis semiteretibus basi subincrassatis $3 \mathrm{~cm}$ longis, interjugis carinatis $2.5-3.5 \mathrm{~cm}$ longis, petiolulis foliolorum lateralium semiteretibus supra sulcatis $0.8 \mathrm{~cm}$ longis terminalibus teretibus $2 \mathrm{~cm}$ longis, foliolis oblongis $8.5(6.5-11) \mathrm{cm}$ longis $3(2.5-4) \mathrm{cm}$ latis basalibus brevioribus, foliolis prope basim acute cuneatam angustatis interdum etiam supra medium angustatis terminalibus a medio basim versus distincte angustatis, apice gradatim acuminatis acumine attenuato obtuso $6 \mathrm{~mm}$ longo 2 vel $3 \mathrm{~mm}$ lato, margine integro, subcoriaceis leavibus supra nitidis infra opacis, nervis sec. utrinque 10-12 nervis prim. prominentibus nervis sec. prominulis nervis tert. conspicuis. Inflorescentiae axillares glomeruliformes dense ramosae pauciflorae $1 \mathrm{~cm}$ diam. Rhaches teretes cum pedicellis teretibus $2-3 \mathrm{~mm}$ longis bracteis bracteolisque triangularibus obtusis $0.5 \mathrm{~mm}$ longis calycibus corollibusque superficie exteriore sparse puberulae. Flores 5-meri circ. $3 \mathrm{~mm}$ longi. Calyx cupuliformis $1 \mathrm{~mm}$ altus lobis triangularibus acutis tubo aequilongis. Petala elliptica apiculo acuto inflexo. Stamina $102 \mathrm{~mm}$ longa filamentis subulatis antheris oblongis. Discus annularis 10-lobus glaber. Pistillum $2 \mathrm{~mm}$ altum ex ovario ovoideo 5-sulcato sparse puberulo et stigmate sessili 5-lobo compositum.

Holotypus: Dusén 15296 (K).

Brazil, Parana, Serra da Prata, Dusén 15296 (Jul. 1914, fl. fem.) (K).

Protium dusenii Swart resembles $P$. widgrenii Engl., from which it differs by the indumentum of its flowers, specially of the ovary, the glomeruliform inflorescences and the less distinct narrowing of the leaflets.

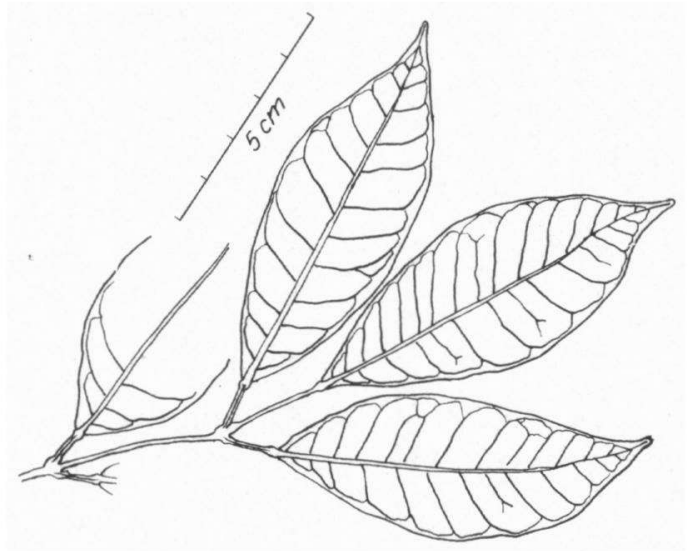

Fig. 3. Protium dusenii Swart-Dusén 15296 (K).

Protium fimbriatum Swart $n$. sp. Arbor usque ad $20 \mathrm{~mm}$ alta. Ramuli robusti angulosi cum petiolis petiolulis rachibusque dense ferrugineo-puberuli. Folia 6 -juga 50 vel $65 \mathrm{~cm}$ longa, petiolis semiteretibus $17.5(15-20) \mathrm{cm}$ longis basi incrassatis adultis transverse 
rimosis, interjugis terete-striatis supra carinatis $3.5-4(2.5-5) \mathrm{cm}$ longis basalibus quam cetera plerumque brevioribus, petiolulis teretibus sulcatis transverse rimosis $5-7.5 \mathrm{~mm}$ longis foliolorum terminalibus teretis $25-30 \mathrm{~mm}$ longis, foliolis ovato-lanceolatis interdum oblongolanceolatis $20(16-24) \mathrm{cm}$ longis $6(5.5-6.5) \mathrm{cm}$ latis terminalibus brevioribus oblongis basalibus minoribus, apice gradatim acuminatis, acumine sublineari obtuso sexies longiora quam latiore $15-20 \mathrm{~mm}$ longo 2.5-3 mm lato, basi rotundo vel subemarginato, margine integro undulato subrepando, subcoriaceis scabridulis supra nitidis subtus opacis, nervis sec. utrinque $13-16$ nervis prim. supra utrinque sulcatis glabris subtus valde prominentibus dense puberulis nervis sec. supra prominulis glabris subtus prominentibus sparse puberulis nervis tert. supra vix prominulis glabris subtus prominentibus pilis nonnullis munitis. Inflorescentiae pseudo-terminalis aut axillaris $15-20 \mathrm{~cm}$ longae ramuli sec. paucis divaricatis usque ad $7.5 \mathrm{~cm}$ longis ramulis tert. nonnullis usque ad $2 \mathrm{~cm}$ longis. Rhaches robustae angulosae dense ferrugineo-puberulae. Pedicelli teretes 2-3 mm longi cum bracteis bracteolisque triangularibus acutis $0.5 \mathrm{~mm}$ longis calycibus corollibusque sparse ferrugineo-puberulis. Flores 5 -meri $3.5 \mathrm{~mm}$ longi. Calyx cupuliformis lobis late triangularius acutis tubo circiter aequilongis. Petala subinduplicativo-valvata oblongo-triangularia acuta carnosa apiculo inflexo incrassato. Stamina 10, in fl. masc. $2 \mathrm{~mm}$ longa, filamenta subulata antheris oblongis duplo longiora. Discus in $\mathrm{fl}$. masc. cum pistillo rudimentario in globulum dense ferrugineo-pubescentem circiter $2 \mathrm{~mm}$ altum confluens, in fl. fem. annularis glaber sed margine distincte et densiuscule fimbriatus. Drupa obliquo-ovoidea et 1-pyrena aut 2- vel 3-loba et 2- vel 3-pyrena, dense ferrugineopuberula basi lobata apice acuta stigmate distincte 5-lobo coronata $2 \mathrm{~cm}$ longa $1-2 \mathrm{~cm}$ diam., mesocarpio crassiusculo carnoso endocarpio tenuo lignoso fragilis.

Holotypus: Krukoff 7024 (U, 23190A).

Brazil, Amazonia, basin of Rio Madeira, near Calama, Krukoff 1308 (Dec. 1931, fruct.) (B, K, NY, U).; id., id., munic, Humayta, on river Livramento, Krukoff 7024 (Oct.-Nov. 1934, fruct. and f. masc.) (K, U).

Protium fimbriatum Swart is near to $P$. apiculatum Swart, but differs by its 6-jugate, 50-65 long leaves and its leaflets with rotundate base and an acumen six times as long as wide, besides a fimbriate disc in the fem. flowers.

Protium firmum Swart n. sp. Arbor $25 \mathrm{~m}$ alta. Ramuli robusti $1 \mathrm{~cm}$ diam. teretes striati juveniles sparse ferrugineo-piloselli, adulti glabrescentes. Folia 6-juga interdum 7-juga, 60-65 cm longa, petiolis prope basim incrassatis semiteretibus $18 \mathrm{~cm}$ longis cum rhachibus petiolulisque pilis patentibus sed brevibus ferruginea obtectis, interjugis teretibus carinatis $5-6 \mathrm{~cm}$ longis basalibus quam cetera brevioribus, petiolulis teretibus canaliculatis adultis transverse rimosis $10-15$ $\mathrm{mm}$ longis foliolorum terminalium teretibus $35 \mathrm{~mm}$ longis, foliolis lanceolatis vel lanceolata-oblongis nonnunquam basim versus angu- 


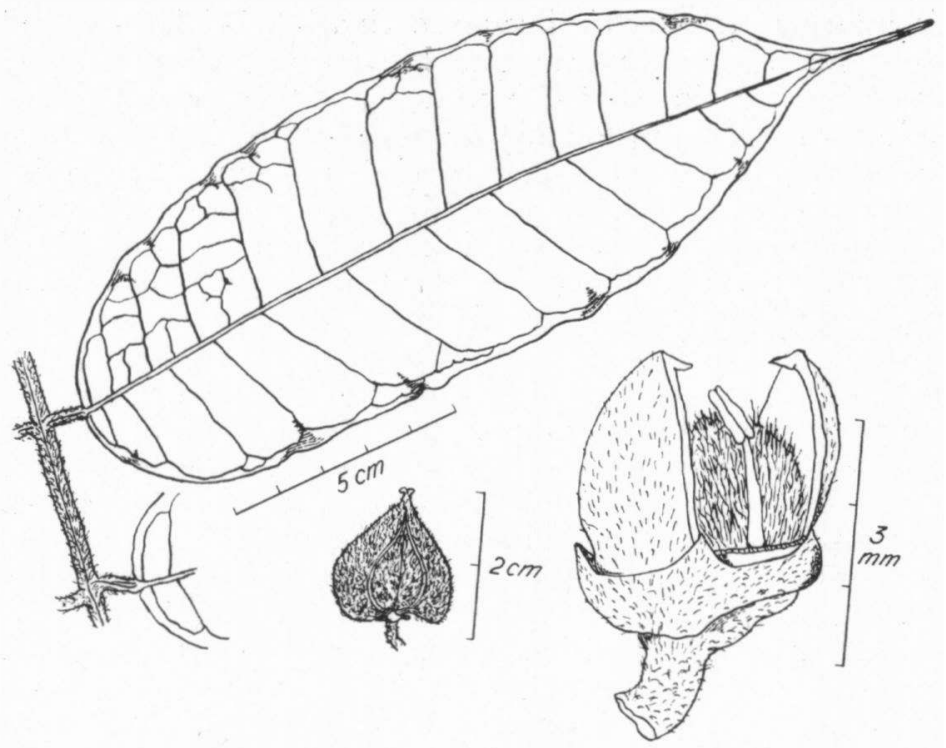

Fig. 4. Protium fimbriatum Swart-Krukoff 7024 (U).

statis $21(18-25) \mathrm{cm}$ longis $6(5-7) \mathrm{cm}$ latis sed foliolis terminalibus et foliolis jugi apicalis et jugi basalis minoribus $16 \mathrm{~cm}$ longis $5 \mathrm{~cm}$ latis, apice gradatim acuminatis, acumine attenua to obtuso longitudine latitudine aequantibus $5 \mathrm{~mm}$ longo, basi acute-cuneato, margine integro, subcoriaceis laevibus glabris supra nitidis subtus opacis, nervis sec. utrinque 14-15, nervis prim. et sec. prominentibus nervis tert. prominulis omnibus glabris costis facie inferiore sparse pilosellis tamen exceptis. Inflorescentiae in axillis solitariae 5-10 cm longae pedunculis robustis ramulis sec. paucis ad $0.5 \mathrm{~cm}$ longis. Rhaches robustae teretestriatae cum bracteis bracteolisque triangularibus $0.5 \mathrm{~mm}$ longis pedicellis teretibus $0.5-1.5 \mathrm{~mm}$ longis calycibus corollibusque facie exteriore sparse minute ferrugineo-pubsecentae. Flores in glomerula structura dichasialia congesti 5 -meri $3 \mathrm{~mm}$ longi. Calyx cupuliformis $1 \mathrm{~mm}$ altus lobis late triangularibus acutis tube fere aequilongis. Petala elliptico-ovata subinduplicativo-valvata subcarnosa apice acuta inflexo-apiculata. Stamina 10 in $\mathrm{fl}$. fem. $1.5 \mathrm{~mm}$ longa, filamenta subulata antheris oblongis bis longiora. Discus in $\mathrm{fl}$. fem. annularus sparse puberulus margine fimbriatus $0.5 \mathrm{~mm}$ altus. Pistillum in $\mathrm{fl}$. fem. densiuscule adpresse ferrugineo-puberulum 1.5 altum ovario ovoideo 5-lobo 5-loculari in stigma sessile 5-lobum attenuato.

Holotypus: Krukoff 7142 (U, 23315A).

Brazil, Amazonas, munic. Humayta, on plateau between Rio Livramento and Rio Ipixuna, Krukoff 1742 (Nov. 1934, fl. fem.) (K, NY, U).

Protium firmum Swart shows conformity with Protium apiculatum Swart, but the latter differs by the smaller, usually 3-jugate leaves, by the smaller, relatively broader and distinctly thinner leaflets with a mu- 
cronulate acumen twice as long as wide and by its much longer subterminal inflorescences.

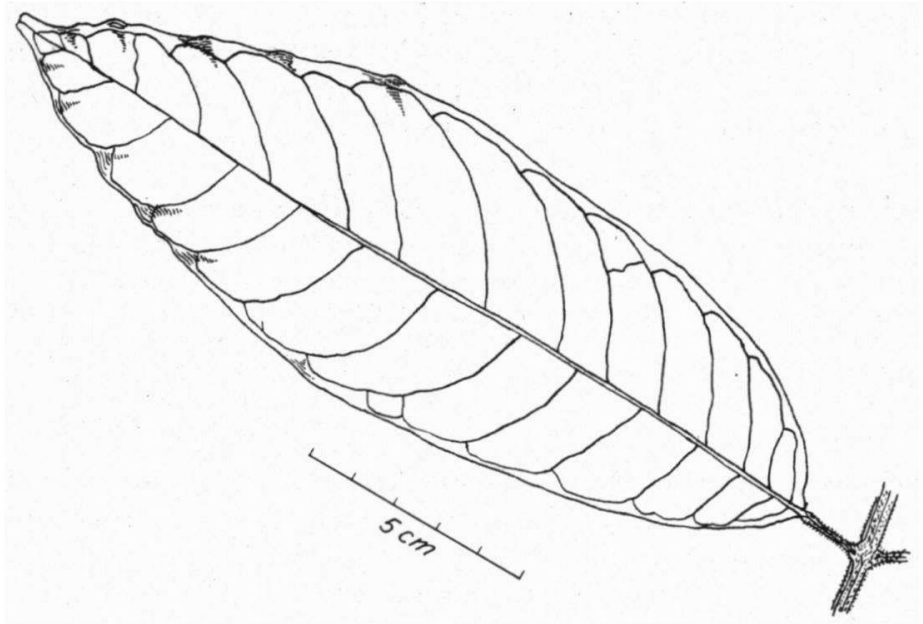

Fig. 5. Protium firmum Swart-Krukoff 7142 (U).

Protium glaziovii Swart n. sp. Arbor?. Ramuli robustini teretes luridi minute piloselli. Folia 3-juga interdum 4-juga $12.5 \mathrm{~cm}$ usque ad $17.5 \mathrm{~cm}$ longa, petiolis robustis semiteretibus $1.75(1-2) \mathrm{cm}$ longis cum interjugis petiolulisque costis foliolorum untrinque sparse et minutissime puberulis, interjugis teretibus supra carinatis 2.25 (1.75$2.75) \mathrm{cm}$ longis, petiolulis semiteretibus sulcatis utroque extumo subincrassatis $0.5 \mathrm{~cm}$ longis sed foliolorum terminalibus $1-1.5 \mathrm{~cm}$ longis, foliolis oblongis $6(4.5-7) \mathrm{cm}$ longis $3(2-3) \mathrm{cm}$ latis terminalibus basim versus paulo angustatis lateralibus non angustatis aut apicem versus paulo angustatis basalibus brevioribus, apicem gradatim acuminatis, acumine attenuato obtuso bis longiore quam latiore $6 \mathrm{~mm}$ longo $3 \mathrm{~mm}$ lato, basi cuneatis, margine integro, pergamentaceis laevibus glabris supra subopacis subtus subnitidis, nervis sec. utrinque 11 , nervis prim. prominentibus nervis sec. prominulis nervis tert. vix distinguendis. Inflorescentiae axillares laxe breviter ramosae petiolis foliorum suffulcientium usque ad bis longiores, rhachibus teretibus striatis sparse puberulis pedunculis usque ad $7 \mathrm{~cm}$ longis ramulis sec. ad $1 \mathrm{~cm}$ longis. Pedicelli teretes $1-2 \mathrm{~mm}$ longi cum bracteis bracteolisque triangularibus acutis $0.4 \mathrm{~mm}$ longis calycibusque densiuscule puberuli. Flores 4-meri $3 \mathrm{~mm}$ longi. Calyx cupuliformis 1 $\mathrm{mm}$ altus lobis triangularibus acutis tubo aequilongis. Petala oblongotriangularia glabra apice acuto incrassato apiculo inflexo munito. Stamina 8 filamentis subulatis $1 \mathrm{~mm}$ longis antheris oblongis $0.75 \mathrm{~mm}$ longis. Discus annularus 8-lobus glaber. Pistillum in $\mathrm{fl}$. masc. $1 \mathrm{~mm}$ altum ovario globoso 4-lobo dense puberulo stylo $0.5 \mathrm{~mm}$ longo stigmate 4-lobo coronato.

Holotypus: Glaziou $19014(\mathrm{~K})$.

BraziL, Rio de Janeiro, Glaziou 19014 (1892, fl. masc.) (B, G, K, LE, US). 
Protium glaziovii Swart is in regard to the indumentum of its vegetative parts similar to $P$. nicaraguense Swart and to $P$. hostmannii Engl., but the fromer differs by its leaves being twice as large and by the acumen of the leaflets being 5-times as long as wide, and the latter species differs by its mostly 2-jugate leaves with larger leaflets and by its inflorescence at most as long as the petiole.

Protium hostmannii Engl. var. brasiliense Swart n. var. Folia 1-juga, petiolis interjugis petiolulisque minutissime puberulis, foliolis subcoriaceis nervis sec. vix prominulis acumine attenuato instructis.

Holotypus: G. A. Black 48-3613 (IAN).

Brazil, Amazonia, Para, Souve, Ilha do Marajo, G. A. Black 48-3613 (1948, fruct. Sept.) (IAN).

Protium paraense Swart $n$. sp. Arbor circ. $10 \mathrm{~m}$ alta. Ramuli graciles $2-3 \mathrm{~mm}$ diam. teretes striati luridi vel castanei, juveniles densiuscule longiuscule pilosi.

Folia 3- vel 5-juga plerumque 4-juga $20(17-21) \mathrm{cm}$ longa, petiolis semiteretibus 2 (1.5-2) cm longis cum interjugis semiteretibus carinatis $2.5(2.25-3) \mathrm{cm}$ longis petiolulisque densiuscule pilosellis pilis brevibus cum pilis aliquibus longioribus deciduis mixtis, petiolulis foliolorum lateralium teretibus sulcatis $2-3 \mathrm{~mm}$ longis foliolorum terminalibus teretibus $15 \mathrm{~mm}$ longis, foliolis lanceolatis vel oblongolanceolatis $8.5(8-9.5) \mathrm{cm}$ longis $2.25(2-2.5) \mathrm{cm}$ latis terminalibus paulo brevioribus basim versus distincte angustatis lateralibus interdum apicem versus paulo angustatis basalibus brevioribus, apice sensim in acumen lineare obtusum $10 \mathrm{~mm}$ longum $2 \mathrm{~mm}$ latum attenuatis, basi acute cuneatis, margine integro undulato, chartaceis utrinque laevibus opacis, nervis sec. utrinque 12 vel 14 nervis prim. utrinque prominentibus nervis sec. supra conspicuis infra prominulis nervis tert. supra inconspicuis infra conspicuis nervis prim. et sec. densiuscule pilosellis. Inflorescentiae axillares glomeruliformes $1 \mathrm{~cm}$ diam. multiflori, rhachibus teretibus cum pedicellis teretibus striatis $1-1.5 \mathrm{~mm}$ longis bracteis bracteolisque triangularibus acutis $0.5 \mathrm{~mm}$ longis densiuscule pilosellis. Flores 4 -meri $3 \mathrm{~mm}$ longi glabri. Calyx cupuliformis $1 \mathrm{~mm}$ altus lobis triangularibus acutis tubo longioribus. Petala induplicativo-valvata ovata acuta apiculo incrassato inflexo. Stamina 8 circ. $2 \mathrm{~mm}$ longa filamentis subulatis antheris ellipticis $0.5 \mathrm{~mm}$ longis. Discus annularis 8-lobus $0.5 \mathrm{~mm}$ altus. Pistillum in fl. masc. disco immersum $0.5 \mathrm{~mm}$ altum, ovario late ovoideo 4-sulcato in stigma sessile 4-lobum attenuato.

Holotypus: Inst. Argon. do Norto Registro 210 (IAN).

Brazil, Amazonia, Belem, Bosque Municipal, Inst. Agron. do Norte Registro 210 (f. masc.) (IAN).

Protium paraense Swart resembles $P$. trifoliolatum Engl., but differs by its 4-jugate leaves with short petiolules and lanceolate leaflets with a narrower acumen and more numerous pairs of sec. nerves. 


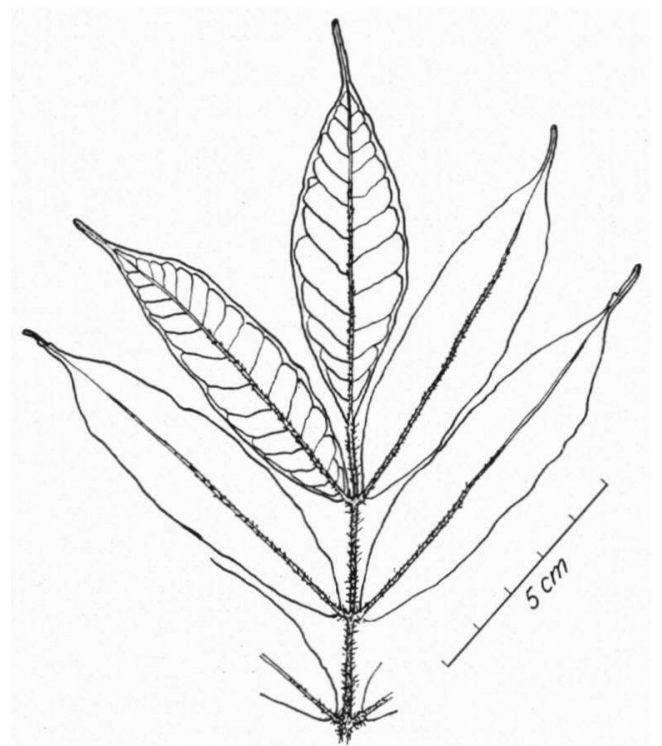

Fig. 6. Protium paraense Swart-IAN Registro 210 (IAN).

Protium piresii Swart n. sp. Arbor. Ramuli teretes striati $3 \mathrm{~mm}$. diam., juveniles sparse ferrugineo-piloselli, demum glabrescentes fulvi. Folia 2- vel 3-juga 15-18 cm longa, petiolis semiteretibus $2.5-3.5 \mathrm{~cm}$ longis cum rhachide petiolulis costis foliolorum facie superiore pilis patentibus densiuscule sed brevissime vestitis, interjugis terete-carinatis apicem versus bisulcatis $2.5-3.5 \mathrm{~cm}$ longis, petiolulis semiteretibus sulcatis utroque extremo subincrassatis 5-6 mm longis sed petiolulis foliolorum terminalium teretibus $20 \mathrm{~mm}$ longis, foliolis oblongis 7.5 (7-9) $\mathrm{cm}$ longis $2(2.75-3.5) \mathrm{cm}$ latis, terminalibus paulo latioris prope basim angustatis, lateralibus plerumque e medio apicem versus paulo angustatis, apicem versus sensim acuminato, acumine sublineari obtuso $10 \mathrm{~mm}$ longo $2 \mathrm{~mm}$ lato, basi cuneatis, margine integris, pergamentaceis laevibus glabris utrinque opacis, nervis sec. utrinque 10, nervis prim. prominentibus supra sparse minutissime pilosis infra glabris nervis sec. glabris supra prominulis infra prominentibus nervis tert. conspicuis. Inflorescentiae axillares e basi ramosae 5-7 cm longae ramulis sec. ad $2 \mathrm{~cm}$ longis rhachibus angularibus cum pedicellis angularibus $1 \mathrm{~mm}$ longis sparse pilosellis. Flores 4-meri $3 \mathrm{~mm}$ longi. Calyx cupuliformis $0.5 \mathrm{~mm}$ altus glaber lobis triangularibus acutis tubo aequilongis. Petala oblongo-triangularia acuta apiculo acuto inflexo instructa glabra margine papillosa. Stamina 8 filamentis subulatis antheris oblongis subaequilongis. Discus annularis glaber 0.5 $\mathrm{mm}$ altus. Pistillum in fl. masc. disco cintum fere $1 \mathrm{~mm}$ altum, ovario ovoideo 4-lobo pilis minutis obtecto, stylo brevi, stigmate 4-lobo.

Holotypus: Pires 2693 (IAN).

Brazru, Amazonia, Para, Belem, Aura. Pires 2693 (1950, f. masc. Nov.) (IAN).

$P$. piresii Swart resembles $P$. hostmannii Engl. but the latter differs 
by its petiole being shorter than the interjugum, by the acumen of the leaflets being only 2- to 3-times as long as wide and by its glomeruliform inflorescences.
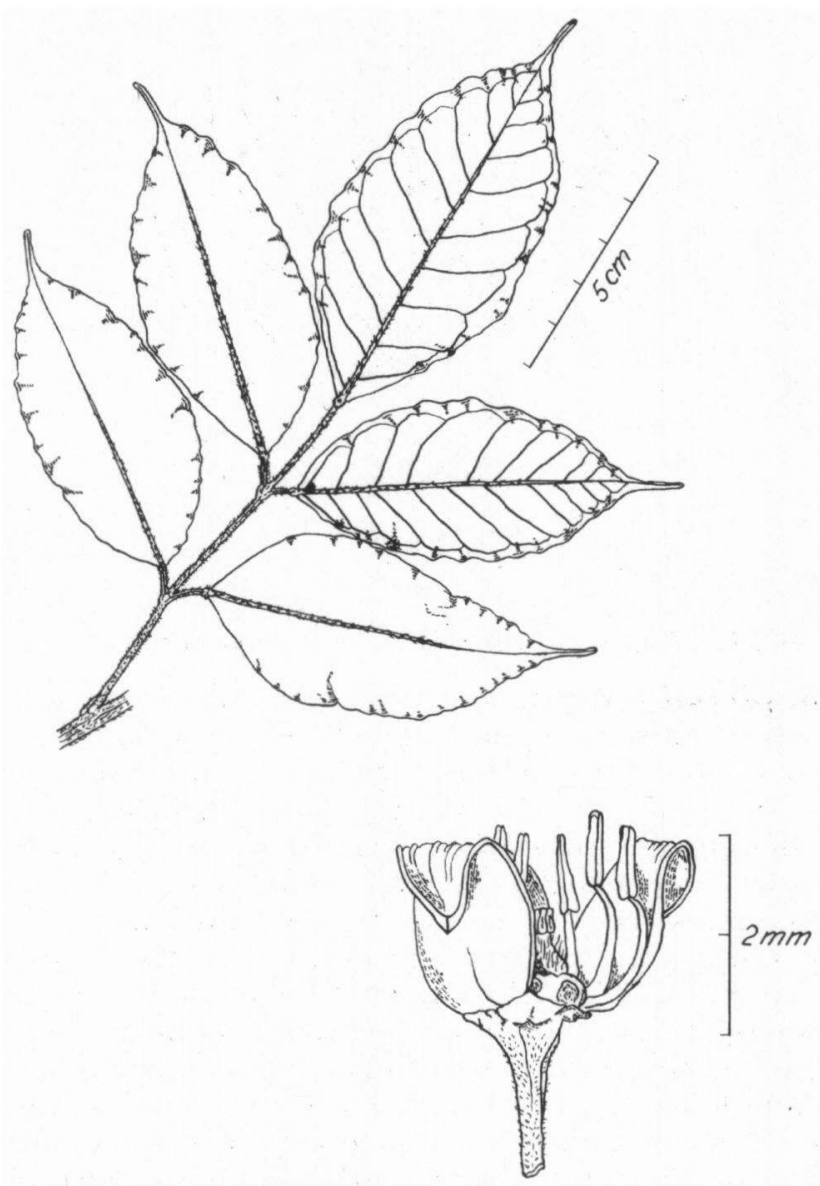

Fig. 7. Protium piresii Swart-Pires 2693 (IAN).

Protium polybotryum (Turcz.) Engl. var. blackii Swart n. var. Folio minora $20-30 \mathrm{~cm}$ longa graciliora, foliolis lanceolatis vel lanceolato-oblongis $9.5(7.5-10.5) \mathrm{cm}$ longis $2.5(2.25-2.75) \mathrm{cm}$ latis. Inflorescentiae longiores quam dimidia pars folii suffulcientis.

Holotypus: G. A. Black 49-8085 (IAN).

Brazir, Amazonia, Para, Belem, Mocambo, G. A, Black, 49-8085 (1949, fruct. July) (IAN).

Protium polybotryum (Turcz.) Engl. var. robustum Swart n. var. Petioli rhaches petioluli pedunculi robusti. Folioli acumen circ. quater longius quam latius, $6-8 \mathrm{~mm}$ longum $1.5-2$ latum. Inflorescentiae in axillis solitariae.

Holotypus: Brit. Guiana Record no. 3140. 


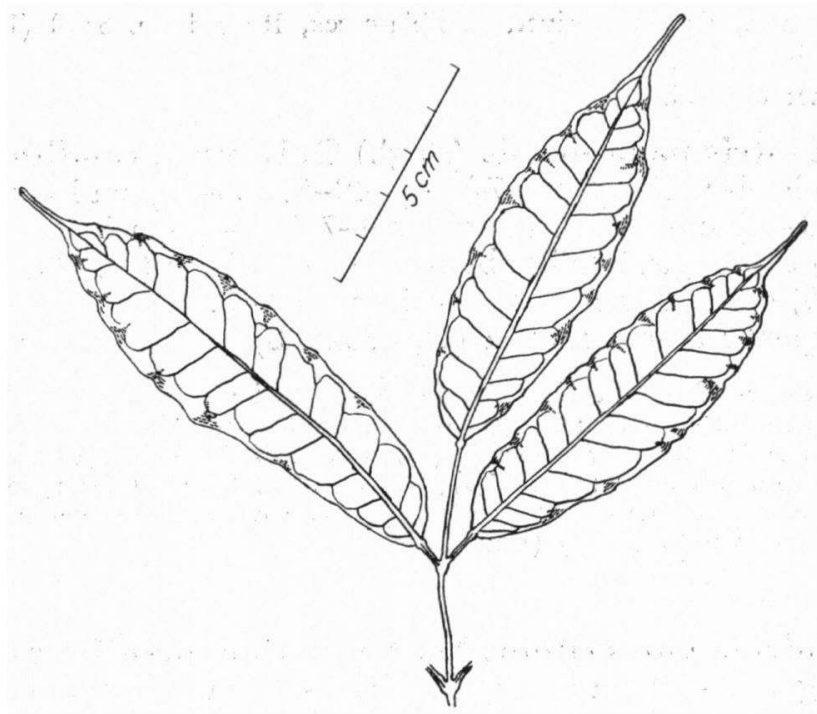

Fig. 8. Protium polybotryum (Turcz.) Engl. var. blackii Swart-Black 49-8085 (IAN).

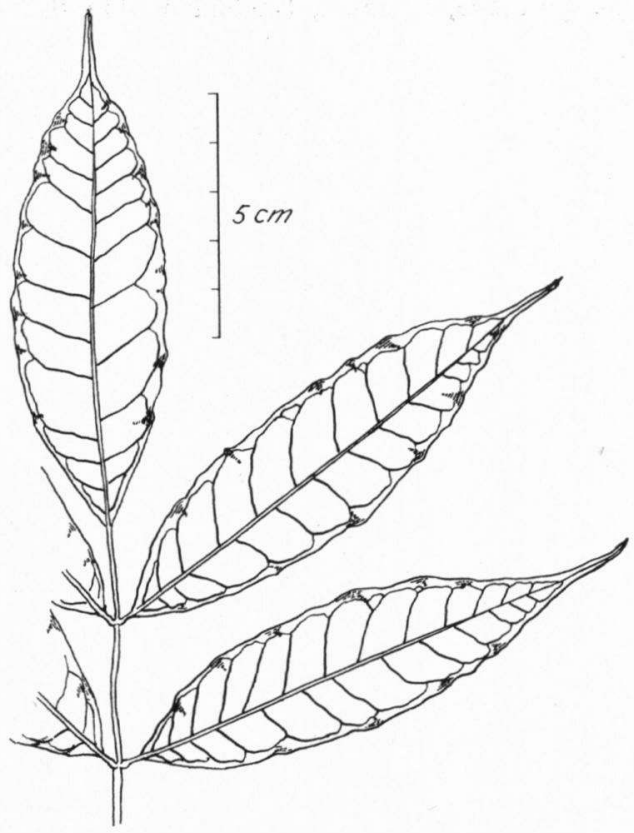

Fig. 9. Tetragastris panamensis (Engl.) O.K. var. grandifolia Swart-Lindeman 5052 (U). 
British Gutana, Demerara-river, Hibibia-creek, Record no. 3140 (1940, fruct. March) (K).

Vern. name: purokai.

Tetragastris panamensis (Engl.) O. K. var. grandifolia Swart n. var. Folio 4- vel 5- (3-vel 7-) juga 40-50 (35-60) cm longa, petiolis 12-16 (10-20) cm longis, interjugis 4-7 (2.5-8) cm longis basalibus 3-5 (2-7) cm longis, foliolis oblongo-lanceolatis $15(9.5-23) \mathrm{cm}$ longis 4.5 (3-6.5) cm latis, nervis sec. utrinque 11-15.

Holotypus: Lindeman 5052 (U, 078233B).

Suriname, slopes of Nassau-mountains, Lanjouw and Lindeman, 2543 (1949) (U); between Suriname-river and Mapane-creek, Lindeman 3659 (1953) (U); id., id. 3736 (1953) (U); id., id. 3748 (1953) (U); id., id. 3776 (1953) (U); id., id. 5038 (1953) (U); id., id. 5039 (1953) (U); id., id. 5052 (1953) (U); type; Moengotapoe, Rikanau, Lindeman, 5905 (1954) (U); between Suriname-river and Mapanecreek, Lindeman 6747 (1954) (U).

Vern. name: salie

Trattinickia burserifolia Mart. var. lindemanii Swart $n$. var. Folia magna $40-50 \mathrm{~cm}$ longa, petiolis $8-11 \mathrm{~cm}$ longis, interjugis 5-8 cm longis, petiolulis $1-2 \mathrm{~cm}$ longis, foliolis usque ad $18 \mathrm{~cm}$ longis interdum apicem versus paulo angustatis acumine bis longiore quam latiore.

Holotypus: Lindeman 4525 (U, 078243B).

Suriname, south of Paramaribo, near Lelydorp, Lindeman 4525 (1953 Aug., fl. buds) (U); Moengo-tapoe, Rikanau, Lindeman 6117 (1954) (U). 